Bull. Korean Math. Soc. 49 (2012), No. 2, pp. 381-394

http://dx.doi.org/10.4134/BKMS.2012.49.2.381

\title{
INSERTION-OF-FACTORS-PROPERTY ON NILPOTENT ELEMENTS
}

\author{
Jineon Baek, Wooyoung Chin, Jiwoong Choi, Taehyun Eom, \\ Young Cheol Jeon, and Yang Lee
}

\begin{abstract}
We generalize the insertion-of-factors-property by setting nilpotent products of elements. In the process we introduce the concept of a nil-IFP ring that is also a generalization of an NI ring. It is shown that if Köthe's conjecture holds, then every nil-IFP ring is NI. The class of minimal noncommutative nil-IFP rings is completely determined, up to isomorphism, where the minimal means having smallest cardinality.
\end{abstract}

\section{Nil-IFP rings}

Throughout this note every ring is an associative ring with identity unless otherwise stated. Given a ring $R$, let $N(R), N_{*}(R)$ and $N^{*}(R)$ denote the set of all nilpotent elements, the prime radical and the upper nilradical (i.e., the sum of all nil ideals) of $R$, respectively. The $n$ by $n$ full (resp. upper triangular) matrix ring over $R$ is denoted by $\operatorname{Mat}_{n}(R)$ (resp. $U_{n}(R)$ ), and $e_{i j}$ 's denote the matrix units. $\mathbb{Z}$ and $\mathbb{Z}_{n}$ denote the ring of integers and the ring of integers modulo $n$. The polynomial ring with an indeterminate $x$ over $R$ is denoted by $R[x]$.

A ring $R$ is called reduced if $N(R)=0$. Any reduced $\operatorname{ring} R$ satisfies, with the help of [20, Proposition 1], that $r_{\sigma(1)} r_{\sigma(2)} \cdots r_{\sigma(n)}=0$ for any permutation $\sigma$ of the set $\{1,2, \ldots, n\}$ when $r_{1} r_{2} \cdots r_{n}=0$ for any positive integer $n$ and $r_{i} \in R$. We will use this fact freely. Due to Bell [5], a ring $R$ is called to satisfy the insertion-of-factors-property, or simply called an IFP ring if $a b=0$ implies $a R b=0$ for $a, b \in R$. Narbonne [25] and Shin [27] used the terms semicommutative and $S I$ for the IFP, respectively. Commutative rings are clearly IFP, and reduced rings are IFP by a simple computation. There exist many non-reduced commutative rings (e.g., $\mathbb{Z}_{n^{k}}$ for $n, k \geq 2$ ), and many noncommutative reduced rings (e.g., direct products of noncommutative domains). A ring is usually

Received November 10, 2010; Revised September 7, 2011.

2010 Mathematics Subject Classification. 16N40, 16U80, 16 S36.

Key words and phrases. nilpotent element, IFP ring, nil-IFP ring, NI ring, polynomial ring.

This work was supported by a 2-Year Research Grant of Pusan National University.

(C)2012 The Korean Mathematical Society 
called abelian if each idempotent is central. IFP rings are abelian by a simple computation.

In the present note we apply the IFP onto the set of all nilpotent elements in place of one-sided ideals. Consider the condition:

$$
a b \in N(R) \text { implies } a R b \subseteq N(R),
$$

where $R$ is a ring (possibly without identity) and $a, b \in R$. We first examine a kind of ring that does not satisfy the condition $(*)$. Every $n$ by $n$ full matrix ring cannot satisfy the condition $(*)$ over any ring when $n \geq 2$.

Example 1.1. Let $A$ be any $\operatorname{ring}$ and $M=\operatorname{Mat}_{n}(A)$ for $n \geq 2$. Consider two matrices $a=e_{11}+e_{21}$ and $b=e_{22}$ in $M$. Then $a b=0$ and $a M b$ contains $c=\left(e_{11}+e_{21}\right)\left(e_{12}+e_{22}\right) e_{22}$. However $c^{k}=e_{12}+e_{22}$ for all $k \geq 1$, entailing that $a M b \nsubseteq N(M)$.

In the following we see relations between IFP rings and rings satisfying the condition $(*)$.

Note. (1) IFP rings satisfy the condition $(*)$. Let $R$ be an IFP ring and $a, b \in R$. Set $a b \in N(R)$ and say $(a b)^{k}=0$. Since $R$ is IFP, $0=a b a b \cdots a b=$ $a R b a R b \cdots a R b=(a R b)^{k}$ and so $a R b \subseteq N(R)$.

(2) Let $R=U_{2}(D)$ for a domain $D$ and $A=\left(a_{i j}\right), B=\left(b_{i j}\right) \in R$. Assume $A B \in N(R)$. Then $a_{11} b_{11}=0$ and $a_{22} b_{22}=0$, entailing that $a_{11} D b_{11}=0$ and $a_{22} D b_{22}=0$. This yields $A R B \subseteq\left(\begin{array}{cc}0 & D \\ 0 & 0\end{array}\right)$. Thus $R$ satisfies the condition (*), but not IFP since it is non-abelian.

Based on the preceding note, a ring $R$ will be called nil-IFP if $R$ satisfies the condition $(*)$.

Next we consider another kind of nil-IFP ring. Marks [23] called a ring $R$ (possibly without identity) $N I$ if $N^{*}(R)=N(R)$. This definition implies that nil rings are NI and that NI rings are clearly nil-IFP. Hong et al. [12, Corollary 13 ] proved that a ring $R$ is NI if and only if every minimal strongly prime ideal of $R$ is completely prime. By the definition we have that a ring $R$ is NI if and only if $N(R)$ forms an ideal if and only if $R / N^{*}(R)$ is reduced. Let $U=U_{n}(R)$ over a ring $R$. Then $N(U)=\left\{m=\left(m_{i j}\right) \in U \mid m_{i i} \in N(R)\right.$ for all $\left.i\right\}$ and $N^{*}(U)=\left\{m=\left(m_{i j}\right) \in U \mid m_{i i} \in N^{*}(R)\right.$ for all $\left.i\right\}$. So $U / N^{*}(U) \cong \oplus_{j=1}^{n} R_{i}$, where $R_{i}=R / N^{*}(R)$ for all $j$. This implies that $R$ is NI if and only if so is $U[14$, Proposition 4.1(1)]. IFP rings are NI by a simple computation but the converse need not hold through $U_{n}(D)$ with $D$ a domain and $n \geq 2$.

First we introduce a lemma which will make our approach to the nature of nil-IFP rings much easier.

Lemma 1.2. For a ring $R$, the following conditions are equivalent:

(1) $R$ is nil-IFP;

(2) If $a b \in N(R)$, then rbsat $\in N(R)$ and rasbt $\in N(R)$ for all $r, s, t \in R$;

(3) If $a \in N(R)$, then ras $\in N(R)$ for all $r, s \in R$; 
(4) If $a \in N(R)$, then $r a \in N(R)$ for all $r \in R$;

(5) If $a \in N(R)$, then ar $\in N(R)$ for all $r \in R$.

Proof. (1) $\Rightarrow(2)$ Let $R$ be nil-IFP. Then we have the following series of assertions: $a b \in N(R) \Rightarrow b a \in N(R) \Rightarrow b s a \in N(R)$ for all $s \in R \Rightarrow s a b \in N(R) \Rightarrow$ satrb $\in N(R)$ for all $r, t \in R \Rightarrow$ rbsat $\in N(R)$. The same computation gives rasbt $\in N(R)$, using $b a \in N(R)$ in place of $a b \in N(R)$.

$(2) \Rightarrow(3)$ It's obvious.

$(3) \Rightarrow(4)$ and $(4) \Rightarrow(5)$ are clear.

$(5) \Rightarrow(1)$ If $a b \in N(R)$, then $b a \in N(R)$ and so $b a R \subseteq N(R)$. Hence $a R b \subseteq$ $N(R)$.

Recall that the center of a ring $R$ is $Z(R)=\{c \in R \mid c r=r c$ for all $r \in R\}$.

Proposition 1.3. (1) The class of nil-IFP rings is closed under subrings (possibly without identity).

(2) If $R$ is a ring such that $N(R) \subseteq Z(R)$, then $R$ is nil-IFP.

(3) Let $R$ be a nil-IFP ring. If $r \in R$ is invertible and $a_{1}, a_{2}, \ldots, a_{n} \in N(R)$ for $n \geq 1$, then $r+a_{1}+a_{2}+\cdots+a_{n}$ is invertible.

Proof. (1) Let $S$ be a subring of a nil-IFP ring $R$. Then $N(S)=N(R) \cap S$. Let $a b \in N(S)$. Then $a b \in N(R)$, so $a R b \subseteq N(R)$. This yields $a S b \subseteq N(S)$, entailing that $S$ is nil-IFP.

(2) Let $a \in N(R)$. If $N(R) \subseteq Z(R)$, then ar $\in N(R)$ for each $r \in R$, entailing that $R$ is nil-IFP by Lemma 1.2 .

(3) Let $R$ be nil-IFP. Note that if $a \in N(R)$, then $1+a$ is invertible. Let $r \in R$ be invertible and $e_{0}$ be the inverse of $r$. Then $e_{0}\left(r+a_{1}+a_{2}+\cdots+a_{n}\right)=$ $1+e_{0} a_{1}+e_{0} a_{2}+\cdots+e_{0} a_{n}$. Since $R$ is nil-IFP, $e_{0} a_{1} \in N(R)$ by Lemma 1.2. Say that $e_{1} \in R$ is the inverse of $1+e_{0} a_{1}$. Then $e_{1}\left(1+e_{0} a_{1}+e_{0} a_{2}+\cdots+e_{0} a_{n}\right)=$ $1+e_{1} e_{0} a_{2}+e_{1} e_{0} a_{3}+\cdots+e_{1} e_{0} a_{n}$. Next we consider the nilpotent $e_{1} e_{0} a_{2}$ (by Lemma 1.2) and the invertible $1+e_{1} e_{0} a_{2}$. Then inductively we can obtain

$$
\begin{aligned}
& e_{n} e_{n-1} \cdots e_{1} e_{0}\left(r+a_{1}+a_{2}+\cdots+a_{n}\right) \\
= & e_{n} e_{n-1} \cdots e_{1}\left(1+e_{0} a_{1}+e_{0} a_{2}+\cdots+e_{0} a_{n}\right) \\
= & \cdots=e_{n}\left(1+e_{n-1} \cdots e_{1} e_{0} a_{n}\right)=1,
\end{aligned}
$$

where $e_{i} \in R$ is the inverse of $1+e_{i-1} \cdots e_{1} e_{0} a_{i}$ for $1 \leq i \leq n$, noting that $e_{i-1} \cdots e_{1} e_{0} a_{i} \in N(R)$ for all $i$ by Lemma 1.2. Whence $r+a_{1}+a_{2}+\cdots+a_{n}$ is invertible.

The class of nil-IFP rings is closed under subrings by Proposition 1.3(1), but it is not closed under factor rings as follows. For example, let $R$ be the ring of quaternions with integer coefficients. Then $R$ is a domain and so nil-IFP. However for any odd prime integer $q$, the ring $R / q R$ is isomorphic to $\operatorname{Mat}_{2}\left(\mathbb{Z}_{q}\right)$ by the argument in $[10$, Exercise $2 \mathrm{~A}]$. But $\operatorname{Mat}_{2}\left(\mathbb{Z}_{q}\right)$ is not nil-IFP by Example 1.1 . 
Proposition 1.4. Let $R$ be a ring and $I$ be a proper ideal of $R$. If both $R / I$ and $I$ (as a ring without identity) are nil-IFP, then so is $R$.

Proof. Suppose that both $R / I$ and $I$ (as a ring without identity) are nil-IFP. Write $\bar{r}=r+I$ for $r \in R$. Let $a \in N(R)$. Then $\bar{a} \in N(R / I)$. Since $R / I$ is nil-IFP, Lemma 1.2 implies that $\overline{r a} \in N(R / I)$ for any $r \in R$. Hence $(r a)^{k} \in I$ for some positive integer $k$ and so $(r a)^{k} r \in I$. Since $a \in N(R), a^{m}=0$ for some positive integer $m$. Then we have $(r a)^{k} r a^{m}(r a)^{k}=0 \in N(I)$. Note that $(r a)^{k} r a^{m-1}, a(r a)^{k} \in I$. Since $I$ is nil-IFP, we get

$$
\left((r a)^{k} r a^{m-1}\right)\left((r a)^{k} r\right)\left(a(r a)^{k}\right) \in N(I)
$$

and

$$
\left((r a)^{k} r a^{m-2}\right)\left(a(r a)^{k} r\right)\left(a(r a)^{k}\right)=\left((r a)^{k} r a^{m-2}\right)\left(a(r a)^{2 k+1}\right) \in N(I) .
$$

Note that $(r a)^{k} r a^{m-2}, a(r a)^{2 k+1} \in I$. Since $I$ is nil-IFP, we get

$$
\left((r a)^{k} r a^{m-2}\right)\left((r a)^{k} r\right)\left(a(r a)^{2 k+1}\right) \in N(I)
$$

and

$$
\left((r a)^{k} r a^{m-3}\right)\left(a(r a)^{k} r\right)\left(a(r a)^{2 k+1}\right)=\left((r a)^{k} r a^{m-3}\right)\left(a(r a)^{3 k+2}\right) \in N(I) .
$$

Repeating this process, we eventually obtain

$$
\left((r a)^{k} r a\right)\left((r a)^{k} r\right)\left(a(r a)^{(m-1) k+(m-2)}\right)=(r a)^{(m+1) k+m} \in N(I) .
$$

Thus $r a \in N(I) \subseteq N(R)$ and so $R$ is nil-IFP by Lemma 1.2 .

Corollary 1.5. (1) Let $R$ be a ring and $I$ be a nil ideal of $R$. Then $R$ is nil-IFP if and only if so is $R / I$.

(2) Let e be a central idempotent of a ring $R$. Then $R$ is nil-IFP if and only if $e R$ and $(1-e) R$ are both nil-IFP.

Proof. (1) Let $R$ be nil-IFP and $a+I \in N(R / I)$. Then since $I$ is nil, we have $a \in N(R)$. Next since $R$ is nil-IFP, $a r \in N(R)$ for all $r \in R$. This yields ar $+I \in N(R / I)$ and so $R / I$ is nil-IFP by Lemma 1.2. Conversely assume that $R / I$ is nil-IFP. Since $I$ is nil, $I$ is nil-IFP clearly. Then $R$ is nil-IFP by Proposition 1.4.

(2) The necessity comes from Proposition 1.3(1) since $e R$ and $(1-e) R$ are subrings of $R$. For the converse, use $e R \cong R /(1-e) R$ and Proposition 1.4.

For the proof of the "if part" of Corollary 1.5(1), we can use directly the condition that $I$ is nil, without using Proposition 1.4. Let $a \in N(R)$. Then since $R / I$ is nil-IFP, ar $+I \in N(R / I)$ for all $r \in R$ by Lemma 1.2. But $I$ is nil and so ar $\in N(R)$, concluding that $R$ is nil-IFP by Lemma 1.2 .

A ring $R$ is called directly finite if $a b=1$ implies $b a=1$ for $a, b \in R$. Example 1.1 shows that there exists a directly finite ring but not nil-IFP. $N I$ rings are directly finite by [14, Proposition 2.7].

Proposition 1.6. Nil-IFP rings are directly finite. 
Proof. Let $R$ be a nil-IFP ring. Suppose $a b=1$ for $a, b \in R$. Then $a b a=a$, so $a(b a-1)=0 \in N(R)$. Since $R$ is a nil-IFP ring, $a b(b a-1)=b a-1 \in N(R)$ by Lemma 1.2. Say $(b a-1)^{n}=0$ for $n \geq 1$. Then

$$
\begin{aligned}
0 & =(b a-1)^{n}=(b a-1)(b a-1)^{n-1} \\
& =b a(b a-1)^{n-1}-(b a-1)^{n-1}=-(b a-1)^{n-1}=\cdots=(-1)^{n-1}(b a-1),
\end{aligned}
$$

entailing $b a=1$. Thus $R$ is directly finite.

We have another proof of Proposition 1.6 by applying [9, Proposition 5.5]. Let $R$ be a nil-IFP ring and assume on the contrary that $R$ is not directly finite. Then $R$ contains an infinite set of matrix units

$$
\left\{e_{11}, e_{12}, e_{13}, \ldots, e_{21}, e_{22}, e_{23}, \ldots\right\}
$$

by [9, Proposition 5.5]. Take $a=e_{11}+e_{21}, b=e_{22} \in R$ as in Example 1.1 , noting $a b=0$. Since $R$ is nil-IFP, $e_{12}+e_{22}=a\left(e_{12}+e_{22}\right) b \notin N(R)$, a contradiction.

A ring $R$ (possibly without identity) is called 2-primal if $N_{*}(R)=N(R)$, according to Birkenmeier et al. [6]. It is obvious that $R$ is 2-primal if and only if $R / N_{*}(R)$ is reduced. It is also easy to see that 2-primal rings are NI, but the converse need not hold by Marks [23, Example 2.2]. The index of nilpotency of a nilpotent element $b$ in a ring $R$ is the least positive integer $n$ such that $b^{n}=0$. The index of nilpotency of a subset $S$ of $R$ is the supremum of the indices of nilpotency of all nilpotent elements in $S$. If such a supremum is finite, then $S$ is said to be of bounded index of nilpotency.

Proposition 1.7. Suppose that a ring $R$ is of bounded index of nilpotency. Then the following conditions are equivalent:

(1) $R$ is nil-IFP;

(2) $R$ is 2-primal;

(3) $R$ is NI.

Proof. It suffices to show $(1) \Rightarrow(2)$. We apply the proof of [14, Proposition 1.4]. Let $R$ be nil-IFP and $a \in N(R)$. Then $a R \subseteq N(R)$ by Lemma 1.2. Since $R$ is of bounded index of nilpotency, either $a R=0$ or $a R$ contains a nonzero nilpotent ideal of $R$ by Levitzki [11, Lemma 1.1] or Klein [18, Lemma 5]. Thus $a R \subseteq P$ for every prime ideal $P$ of $R$, entailing $a \in N_{*}(R)$. This implies that $R$ is 2-primal.

The following is an immediate consequence of Proposition 1.7.

Corollary 1.8. Every finite nil-IFP ring is 2-primal (hence NI).

If a ring $R$ is nil-IFP but not NI, then $N(R)$ is not closed under addition by Lemma 1.2. So if such a ring exists, then it must contain an infinite subring as follows (otherwise, $R$ is NI by Proposition 1.7). In the following we also see a necessary condition for Köthe's conjecture (i.e., the upper nilradical contains all nil left ideals) to hold. 
Proposition 1.9. (1) Let $R$ be a nil-IFP ring but not NI. Then there exists a countably infinite subring of $R$ which is nil-IFP but not NI.

(2) NI and nil-IFP properties are equivalent if and only if NI and nil-IFP properties are equivalent for countably infinite rings.

(3) If Köthe's conjecture holds, then every nil-IFP ring is NI.

Proof. (1) Since $R$ is nil-IFP but not NI, there exist two nilpotent elements $a$ and $b$ of $R$ such that $a+b$ is not nilpotent. Consider the subring $S$ of $R$ generated by $a$ and $b$. Then $S=\mathbb{Z}[a, b]$ with $a^{m}=b^{m}=0$ for some $m \geq 2$. Since $a+b$ is not nilpotent, $S$ is countably infinite. $S$ is nil-IFP by Lemma $1.3(1)$, but not NI by the fact $a+b \notin N(S)$.

(2) is an immediate consequence of (1).

(3) Suppose that Köthe's conjecture holds. Let $R$ be a nil-IFP ring and $a, b \in N(R)$. It suffices to show $a+b \in N(R)$. $r a, s b \in N(R)$ for all $r, s \in R$ by Lemma 1.2. Let $I=\{r a \mid r \in R\}$ and $J=\{s b \mid s \in R\}$. Then clearly $I$ and $J$ are left nil ideals of $R$. Since Köthe's conjecture holds, $I+J \subseteq N(R)$, so $a+b \in N(R)$. Thus, $N(R)$ is an ideal of $R$, entailing that $R$ is NI.

As a contraposition of Proposition 1.9(3), we can say that if the implication from nil-IFP to NI is proper, then Köthe's conjecture need not hold.

In the following we see some information about the set of nilpotent elements in nil-IFP rings.

Proposition 1.10. Let $R$ be a nil-IFP ring and $a, b \in N(R)$ with $a^{n}=b^{m}=0$ for some positive integers $m, n$.

(1) Suppose that $i_{1}, i_{2}, j_{1}, j_{2}$ are nonnegative integers such that $i_{1}+i_{2} \geq n$ and $j_{1}+j_{2} \geq m$. Then $\left(a^{i_{1}}+b^{j_{1}}\right)\left(a^{i_{2}}+b^{j_{2}}\right) \in N(R)$.

(2) If $a^{2}=b^{2}=0$, then $a+b \in N(R)$.

(3) $a^{\alpha}+b^{\beta} \in N(R)$ for $\alpha, \beta \in \mathbb{Z}$ such that $2 \alpha \geq n$ and $2 \beta \geq m$.

(4) Let $r \in Z(R)$ such that $r a b+b a=0$. Then $a+b \in N(R)$.

Proof. (1) First we have $\left(a^{i_{1}}+b^{j_{1}}\right)\left(a^{i_{2}}+b^{j_{2}}\right)=a^{i_{1}} b^{j_{2}}+b^{j_{1}} a^{i_{2}}$ from the condition that $i_{1}+i_{2} \geq n$ and $j_{1}+j_{2} \geq m$. Moreover

$$
\left(\left(a^{i_{1}}+b^{j_{1}}\right)\left(a^{i_{2}}+b^{j_{2}}\right)\right)^{k}=\left(a^{i_{1}} b^{j_{2}}+b^{j_{1}} a^{i_{2}}\right)^{k}=\left(a^{i_{1}} b^{j_{2}}\right)^{k}+\left(b^{j_{1}} a^{i_{2}}\right)^{k}
$$

for every positive integer $k$. Since $a, b \in N(R),\left(a^{i_{1}} b^{j_{2}}\right)^{k}$ and $\left(b^{j_{1}} a^{i_{2}}\right)^{k}$ are nilpotent by Lemma 1.2. Thus we also have $\left(\left(a^{i_{1}}+b^{j_{1}}\right)\left(a^{i_{2}}+b^{j_{2}}\right)\right)^{k} \in N(R)$ by Lemma 1.2.

(2) If $a^{2}=b^{2}=0$, then $(a+b)^{2}=(a+b)(a+b) \in N(R)$ by (1), and hence $a+b \in N(R)$.

(3) Since $a^{n}=b^{m}=0,2 \alpha \geq n$, and $2 \beta \geq m$, it follows that $\left(a^{\alpha}+b^{\beta}\right)^{2}=$ $\left(a^{\alpha}+b^{\beta}\right)\left(a^{\alpha}+b^{\beta}\right) \in N(R)$ by $(1)$. Hence $a^{\alpha}+b^{\beta} \in N(R)$.

(4) Since $r a b+b a=0, b a=-r a b$. Then each term of expansion of $(a+$ $b)^{n+m-1}$ can be simplified as $(-r)^{\sigma} a^{k} b^{n+m-1-k}$ where $k$ is an integer such that $0 \leq k \leq n+m-1$ and $\sigma$ is some positive integer. If $k \geq n$, then $a^{k}=0$. If 
$k<n$, then $b^{n+m-1-k}=0$. So each term of the expansion of $(a+b)^{n+m-1}$ is equal to 0 , entailing $(a+b)^{n+m-1}=0$.

Consider $e_{12}, e_{21} \in \operatorname{Mat}_{2}(A)$ over a ring $A$. Then $e_{12}^{2}=e_{21}^{2}=0$ but $e_{12}+e_{21}$ is not nilpotent. So the condition that $R$ is a nil-IFP ring is not superfluous in Proposition 1.10 .

Nil-IFP rings need not be abelian as can be seen by $U_{2}(R)$, over a reduced ring $R$, which is nil-IFP by Proposition 2.3 to follow. The next example illuminates that abelian rings also need not be nil-IFP. Due to Armendariz [3, Lemma 1], Rege et al. [26] called a ring $R$ Armendariz if $a_{i} b_{j}=0$ for all $i$ and $j$ whenever $f(x)=\sum_{i=0}^{m} a_{i} x^{i}, g(x)=\sum_{j=0}^{n} b_{j} x^{j} \in R[x]$ satisfy $f(x) g(x)=0$. Armendariz rings are abelian by the proof of [1, Theorem 6$]$.

Example 1.11. Let $S=\mathbb{C}\{a, b\}$ be the free algebra with non-commuting indeterminates $a, b$ over $\mathbb{C}$, where $\mathbb{C}$ is the field of complex numbers. Let $I$ be an ideal of $S$ generated by $a^{2}$. Set $R=S / I$. We coincide $a, b$ with their images in $R$ for simplicity. Notice that $N(R)$ is the subring of $R$ generated by

$$
\{\alpha a, \beta \text { ara } \mid \alpha, \beta \in \mathbb{C}, r \in R\} .
$$

Since $b a a \in N(R)$ and $b a b a \notin N(R), R$ is not nil-IFP. But $R$ is Armendariz (hence abelian) by [2, Example 4.8] or [7, Examples 9.3].

A ring $R$ is called (von Neumann) regular if for each $a \in R$ there exists $x \in R$ such that $a=a x a$.

Proposition 1.12. Let $R$ be a regular ring. Then the following conditions are equivalent:

(1) $R$ is reduced;

(2) $R$ is $N I$;

(3) $R$ is nil-IFP;

(4) $R$ is 2-primal;

(5) $R$ is abelian;

(6) $R$ is IFP.

Proof. Every regular ring is semiprimitive by [9, Corollary 1.2(c)], entailing that regular NI rings are reduced. Abelian regular rings are reduced by $[9$, Theorem 3.2(c)]. Let $R$ be a nil-IFP ring and let $a \in N(R)$. Since $R$ is regular, there exists $b \in R$ such that $a=a b a$. Notice that $b a$ is an idempotent element of $R$. But Lemma 1.2 implies $b a \in N(R)$ since $R$ is nil-IFP and $a \in N(R)$. Therefore, $b a$ must be zero. Thus, $a=a b a=a 0=0$, entailing that $R$ is reduced.

A ring $R$ is called $\pi$-regular if for each $a \in R$ there exists a positive integer $n$, depending on $a, b \in R$ such that $a^{n}=a^{n} b a^{n}$. The Jacobson radicals of $\pi$-regular rings are nil, comparing with that regular rings are semiprimitive. Regular rings are clearly $\pi$-regular. However the preceding result need not 
hold on $\pi$-regular rings. $U_{n}(D)(n \geq 2$ and $D$ is a division ring) is $\pi$-regular and nil-IFP, but it is neither regular nor reduced.

In the following arguments, we characterize the class of minimal noncommutative nil-IFP rings for the cases of with identity and without identity. The term minimal means having the smallest cardinality. $|S|$ means the cardinality of a set $S$.

Proposition 1.13. Let $R$ be a ring with identity. If $R$ is a minimal noncommutative nil-IFP ring, then $R$ is of order 8 and is isomorphic to $U_{2}\left(\mathbb{Z}_{2}\right)$.

Proof. Let $R$ be a minimal noncommutative nil-IFP ring with identity. Then $|R| \geq 2^{3}$ by $\left[8\right.$, Theorem]. If $|R|=2^{3}$, then $R$ is isomorphic to $U_{2}\left(\mathbb{Z}_{2}\right)$ by $[8$, Proposition]. But $U_{2}\left(\mathbb{Z}_{2}\right)$ is a nil-IFP ring by Proposition 2.3 to follow. This implies that $R$ is of order 8 and is isomorphic to $U_{2}\left(\mathbb{Z}_{2}\right)$.

Next we observe the structure of minimal noncommutative nil-IFP rings without identity.

Example 1.14. Consider the subrings

$$
R_{1}=\left(\begin{array}{cc}
\mathbb{Z}_{2} & \mathbb{Z}_{2} \\
0 & 0
\end{array}\right), R_{2}=\left(\begin{array}{cc}
0 & \mathbb{Z}_{2} \\
0 & \mathbb{Z}_{2}
\end{array}\right) \text {, and } R_{3}=\left(\begin{array}{ccc}
\mathbb{Z}_{2} & 0 & 0 \\
0 & 0 & \mathbb{Z}_{2} \\
0 & 0 & 0
\end{array}\right)
$$

of $U_{2}\left(\mathbb{Z}_{2}\right)$ and $U_{3}\left(\mathbb{Z}_{2}\right)$. Then $R_{i}$ is nil-IFP for $i=1,2,3$ by Proposition $1.3(1)$ and Proposition 2.3 to follow.

Consider the subring

$$
R_{4}=\mathbb{Z}_{2} e_{i i}+\mathbb{Z}_{2} e_{j k} \text { with } i \neq j, i \neq k, j \neq k
$$

of $U_{l}\left(\mathbb{Z}_{2}\right)$ for $l \geq 3$. Then $R_{3}$ is isomorphic to $R_{4}$ with $e_{11} \mapsto e_{i i}$ and $e_{23} \mapsto e_{j k}$.

Given a ring $R, R^{+}$means the additive abelian group $(R,+)$. The Jacobson radical of a ring $R$ is denoted by $J(R)$.

Proposition 1.15. Let $R$ be a ring without identity. If $R$ is a minimal noncommutative nil-IFP ring, then $R$ is of order 4 and is isomorphic to $R_{i}$ for some $i \in\{1,2,3\}$, where $R_{i}$ 's are the rings in Example 1.14.

Proof. Let $R$ be a minimal noncommutative nil-IFP ring without identity. If $|R| \leq 3$, then $R$ must be commutative, and so $|R| \geq 2^{2}$. Then $|R|=2^{2}$ by considering the rings in Example 1.14. Assume that $R$ is nil. Note that $J(R)=N^{*}(R)=R$ and $R$ is nilpotent. If $R^{+}$is cyclic, then $R$ is commutative clearly. If $R^{+}$be non-cyclic, then $R$ is also commutative by [19, Theorem 2.3.3]. Thus $R$ must be non-nil, entailing that $J(R)=0$ or $|J(R)|=2$.

If $J(R)=0$, then $R$ is commutative by applying the proof of [15, Theorem 3.3]. Consequently we get to the only case of $|J(R)|=2$. We also apply the proof of $\left[15\right.$, Theorem 3.3] in this case. $|J(R)|=2$ implies $R / J(R) \cong \mathbb{Z}_{2}$. By [21, Proposition 3.6.2], there exists an idempotent $e \in R$ such that $1+J(R)=$ $e+J(R)$. Then there exists $0 \neq b \in J(R)$ such that $R=\{0, e, b, e+b\}$ and 
$J(R)=\{0, b\}$, where $b^{2}=0$ (if $b^{2} \neq 0$, then $b^{2}=b$, a contradiction). Here we have the following three cases:

$$
(e b=b, b e=0),(b e=b, e b=0), \text { and }(e b=0, b e=0),
$$

noting that if $b e=b=e b$, then $R$ is commutative. Therefore $R$ is isomorphic to one of the rings

$$
R_{1}=\left(\begin{array}{cc}
\mathbb{Z}_{2} & \mathbb{Z}_{2} \\
0 & 0
\end{array}\right),\left(\begin{array}{cc}
0 & \mathbb{Z}_{2} \\
0 & \mathbb{Z}_{2}
\end{array}\right), \text { and }\left(\begin{array}{ccc}
\mathbb{Z}_{2} & 0 & 0 \\
0 & 0 & \mathbb{Z}_{2} \\
0 & 0 & 0
\end{array}\right)
$$

in Example 1.14, through one of the maps

$$
\left(e \mapsto e_{11}, b \mapsto e_{12}\right),\left(e \mapsto e_{22}, b \mapsto e_{12}\right) \text {, and }\left(e \mapsto e_{11}, b \mapsto e_{23}\right) \text {. }
$$

Note that $U_{2}\left(\mathbb{Z}_{2}\right)$ and the rings in Example 1.14 are all NI rings. So we also obtain the following by Propositions 1.13 and 1.15.

Corollary 1.16. Let $R$ be a ring (possibly without identity). Then $R$ is a minimal noncommutative nil-IFP ring if and only if $R$ is a minimal NI ring if and only if $R$ is a minimal 2-primal ring.

\section{Examples of nil-IFP rings}

Let $R$ be an algebra over a commutative ring $S$. Recall that the Dorroh extension of $R$ by $S$ is the ring $R \times S$ with operations $\left(r_{1}, s_{1}\right)+\left(r_{2}, s_{2}\right)=$ $\left(r_{1}+r_{2}, s_{1}+s_{2}\right)$ and $\left(r_{1}, s_{1}\right)\left(r_{2}, s_{2}\right)=\left(r_{1} r_{2}+s_{1} r_{2}+s_{2} r_{1}, s_{1} s_{2}\right)$, where $r_{i} \in R$ and $s_{i} \in S$.

Proposition 2.1. (1) Let $R=\sum_{i \in I} R_{i}$ be a direct sum of rings $R_{i} . \quad R$ is nil-IFP if and only if $R_{i}$ is nil-IFP for each $i \in I$.

(2) Let $R$ be an algebra over a commutative ring $S$. Then the Dorroh extension $D$ of $R$ by $S$ is nil-IFP if and only if so is $R$.

(3) Let $R$ be a ring without identity. Attach an identity to $R$ by considering the ring $R \times \mathbb{Z}$ with the same operations as in Dorroh extension. Then $R \times \mathbb{Z}$ is nil-IFP if and only if so is $R$.

(4) The class of nil-IFP rings is closed under direct limits.

Proof. (1) Let $a=\left(a_{i}\right) \in N(R)$ and $r=\left(r_{i}\right) \in R$. Then there exist positive integers $m, n$ such that $a_{k}=0$ for all $k>m$ and $a_{i}^{n}=0$ for all $i \in I$. Since each $R_{i}$ is nil-IFP, $a_{i} r_{i} \in N\left(R_{i}\right)$ for all $i=1, \ldots, m$ by Lemma 1.2. Then there exists some positive integer $h$ such that $\left(a_{i} r_{i}\right)^{h}=0$ for all $i \in I$. Hence, ar $\in N(R)$ and so $R$ is nil-IFP by Lemma 1.2.

Conversely, let $R$ be nil-IFP and $k \in I$. Suppose that $a_{k} \in N\left(R_{k}\right)$ and $r_{k} \in R_{k}$. Take $a=\left(a_{i}\right), r=\left(r_{i}\right) \in R$ such that $a_{i}=0$ for all $i \in I \backslash\{k\}$. Then obviously $a \in N(R)$. By Lemma 1.2, ar $\in N(R)$ and this yields $a_{k} r_{k} \in N\left(R_{k}\right)$. Thus $R_{k}$ is nil-IFP by Lemma 1.2 .

(2) Let $R$ be a nil-IFP ring. Note $R \cong R \times 0$, so the proper ideal $R \times 0$ of $D$ is nil-IFP as a ring without identity. Since $D /(R \times 0) \cong S$ and $S$ is nil-IFP, $D$ 
is also nil-IFP by Proposition 1.4. Conversely, if $D$ is nil-IFP, then the subring $R$ of $D$ is nil-IFP by Proposition 1.3(1).

(3) The proof is essentially the same as in (2).

(4) Let $D=\left\{R_{i}, \alpha_{i j}\right\}$ be a direct system of nil-IFP rings $R_{i}$ for $i \in I$ and ring homomorphisms $\alpha_{i j}: R_{i} \rightarrow R_{j}$ for each $i \leq j$ satisfying $\alpha_{i j}(1)=1$, where $I$ is a directed partially ordered set. Set $R=\lim R_{i}$ the direct limit of $D$ with $\iota_{i}: R_{i} \rightarrow R$ and $\iota_{j} \alpha_{i j}=\iota_{i}$. Let $a, b \in \vec{R}$. Then $a=\iota_{i}\left(a_{i}\right)$, $b=\iota_{j}\left(b_{j}\right)$ for some $i, j \in I$ and there is $k \in I$ such that $i \leq k, j \leq k$. Define $a+b=\iota_{k}\left(\alpha_{i k}\left(a_{i}\right)+\alpha_{j k}\left(b_{j}\right)\right)$ and $a b=\iota_{k}\left(\alpha_{i k}\left(a_{i}\right) \alpha_{j k}\left(b_{j}\right)\right)$, where $\alpha_{i k}\left(a_{i}\right)$ and $\alpha_{j k}\left(b_{j}\right)$ are in $R_{k}$. Then $R$ forms a ring with $0=\iota_{i}(0)$ and $1=\iota_{i}(1)$.

Next suppose that $a \in N(R)$ and consider ar for $r \in R$. Then $a=\iota_{i}\left(a_{i}\right)$ and $r=\iota_{j}\left(r_{j}\right)$ for some $i, j \in I$. Further, there exists $k \in I$ such that $\alpha_{i k}\left(a_{i}\right) \in$ $N\left(R_{k}\right)$ and $\iota_{k}\left(\alpha_{i k}\left(a_{i}\right)\right)=\iota_{i}\left(a_{i}\right), \iota_{k}\left(\alpha_{j k}\left(r_{j}\right)\right)=\iota_{j}\left(r_{j}\right)$. Since $R_{k}$ is nil-IFP, $\left.\alpha_{i k}\left(a_{i}\right)\right) \alpha_{j k}\left(r_{j}\right) \in N\left(R_{k}\right)$ and so

$$
a r=\iota_{i}\left(a_{i}\right) \iota_{j}\left(r_{j}\right)=\iota_{k}\left(\alpha_{i k}\left(a_{i}\right)\right) \iota_{k}\left(\alpha_{j k}\left(r_{j}\right)\right)=\iota_{k}\left(\alpha_{i k}\left(a_{i}\right) \alpha_{j k}\left(r_{j}\right)\right) \in N(R) .
$$

So $R$ is nil-IFP by Lemma 1.2.

Observing Proposition 2.1(1), one may suspect that the direct product of nil-IFP rings is also nil-IFP. However, this is not the case as follows.

Example 2.2. The construction and computation are according to [13, Examples 1.6], [14, Example 2.5], and [24, Remark, p. 508]. Let $K$ be a field and define $D_{n}=K\left\{x_{n}\right\}$, a free algebra generated by $x_{n}$, with a relation $x_{n}^{n+2}=0$ for each nonnegative integer $n$. Then clearly $D_{n} \cong K[x] /\left(x^{n+2}\right)$, where $\left(x^{n+2}\right)$ is the ideal of $K[x]$ generated by $x^{n+2}$. Next let $R_{n}=\left(\begin{array}{cc}D_{n} & x_{n} D_{n} \\ x_{n} D_{n} & D_{n}\end{array}\right)$ be a subring of the 2 by 2 matrix ring over $D_{n}$. Then every $R_{n}$ is 2 -primal (hence nil-IFP) by the computation in [13, Examples 1.6]. Set $R=\prod_{n=0}^{\infty} R_{n}$ and consider $\left(x_{n} e_{12}\right) \in R$. Then $\left(x_{n} e_{12}\right) \in N(R)$ but $\left(x_{n} e_{12}\right)\left(x_{n} e_{21}\right)=\left(x_{n}^{2} e_{11}\right) \notin N(R)$. Hence, $R$ is not nil-IFP by Lemma 1.2.

The $n$ by $n$ lower triangular matrix ring over a $\operatorname{ring} R$ is denoted by $L_{n}(R)$.

Proposition 2.3. For a ring $R$ and an integer $n \geq 2$, the following conditions are equivalent:

(1) $R$ is nil-IFP;

(2) $U_{n}(R)$ is nil-IFP;

(3) $L_{n}(R)$ is nil-IFP;

(4) $V_{n}(R)=\left\{\left(\begin{array}{cccccc}a_{1} & a_{2} & a_{3} & \cdots & a_{n-1} & a_{n} \\ 0 & a_{1} & a_{2} & \cdots & a_{n-2} & a_{n-1} \\ 0 & 0 & a_{1} & \cdots & a_{n-3} & a_{n-2} \\ \vdots & \vdots & \vdots & \ddots & \vdots & \vdots \\ 0 & 0 & 0 & \cdots & a_{1} & a_{2} \\ 0 & 0 & 0 & \cdots & 0 & a_{1}\end{array}\right) \mid a_{i} \in R\right\}$ is a nil-IFP ring; 

$x^{n}$.

(5) $R[x] /\left\langle x^{n}\right\rangle$ is a nil-IFP ring, where $\left\langle x^{n}\right\rangle$ is the ideal of $R[x]$ generated by

Proof. Note that $I=\left\{b \in U_{n}(R) \mid\right.$ the diagonal entries of $b$ are all zero $\}$ is a nil ideal of $U_{n}(R)$. Moreover $U_{n}(R) / I$ is isomorphic to the direct sum of $n$ copies of $R$. Then the equivalence between the conditions (1) and (2) is obtained by Corollary 1.5 (1) and Proposition 2.1. The proof of the equivalence between the conditions (1) and (3) is similar to the preceding case.

$(2) \Rightarrow(4)$ and $(4) \Rightarrow(1)$ are shown by Proposition $1.3(1)$.

The equivalence between the conditions (4) and (5) are obtained from the well-known fact that $V_{n}(R) \cong R[x] /\left\langle x^{n}\right\rangle$.

Proposition 2.4. Let $R$ and $S$ be rings and ${ }_{R} M_{S}$ an $(R, S)$-bimodule. Then $E=\left(\begin{array}{cc}R & M \\ 0 & S\end{array}\right)$ is nil-IFP if and only if $R$ and $S$ are both nil-IFP.

Proof. Note that $J=\left(\begin{array}{cc}0 & M \\ 0 & 0\end{array}\right)$ is a nil ideal of $E$ and $E / J \cong R \oplus S$. So the proof is obtained by Corollary 1.5(1) and Proposition 2.1.

In fact, we do not know any example of a nil-IFP ring such that $R[x]$ is not nil-IFP.

Proposition 2.5. Suppose that a ring $R$ is of bounded index of nilpotency. Then the following conditions are equivalent:

(1) $R$ is nil-IFP;

(2) $R[x]$ is 2-primal;

(3) $R[x]$ is $N I$;

(4) $R[x]$ is nil-IFP.

Proof. It suffices to show $(1) \Rightarrow(2)$ by Proposition $1.3(1)$. Let $R$ be of bounded index of nilpotency. Then, by Proposition $1.7, R$ is 2-primal if and only if $R$ is nil-IFP. But $R$ is 2-primal if and only if $R[x]$ is 2-primal by [6, Proposition $2.6]$.

Next we consider the nil-IFP condition of Ore extensions. For a ring $R$, a ring endomorphism $\sigma: R \rightarrow R$ and a $\sigma$-derivation $\delta: R \rightarrow R$, the Ore extension $R[x ; \sigma, \delta]$ of $R$ is the ring obtained by giving $R[x]$ the multiplication $x r=\sigma(r) x+\delta(r)$ for all $r \in R$. If $\delta=0$, we write $R[x ; \sigma]$ for $R[x ; \sigma, 0]$ and is called an Ore extension of endomorphism type (also called a skew polynomial ring). While if $\sigma=1$, we write $R[x ; \delta]$ for $R[x ; 1, \delta]$ and is called an Ore extension of derivation type (also called a differential polynomial ring).

Example 2.6. (1) There exists a nil-IFP ring over which the skew polynomial ring need not be nil-IFP. For a domain $D$ let $R=D \oplus D$, then $R$ is reduced (hence nil-IFP). Consider the automorphism $\sigma$ of $R$ defined by $\sigma((s, t))=(t, s)$. Let $R[x ; \sigma]$ be the skew polynomial ring over $R$ by $\sigma$. Consider $(1,0) x$ and $(0,1) x$. Then $((1,0) x(1,0))=0 \in N(R[x ; \sigma])$ but $(0,1) x x(0,1)=(0,1) x^{2} \notin$ $N(R[x ; \sigma])$, entailing that $R[x ; \sigma]$ is not nil-IFP. 
(2) There exists a nil-IFP ring over which the differential polynomial ring need not be nil-IFP. We use the ring and argument in $\left[4\right.$, Example 11]. Let $\mathbb{Z}_{2}[t]$ be the polynomial ring with an indeterminate $t$ over $\mathbb{Z}_{2}$. Then $R=\mathbb{Z}_{2}[t] /\left(t^{2}\right)$ is commutative (hence nil-IFP), where $\left(t^{2}\right)$ is the ideal of $\mathbb{Z}_{2}[t]$ generated by $t^{2}$. Define a derivation $\delta$ on $R$ by $\delta\left(t+\left(t^{2}\right)\right)=1+\left(t^{2}\right)$. Then $R[x ; \delta] \cong \operatorname{Mat}_{2}\left(\mathbb{Z}_{2}\left[y^{2}\right]\right)$ which is not nil-IFP by example 1.1.

According to Antoine [2, Definition 2.3], a ring $R$ is called nil-Armendariz if $a b \in N(R)$ for every coefficient $a$ of $f(x)$ and every coefficient $b$ of $g(x)$ whenever $f(x), g(x) \in R[x]$ satisfy $f(x) g(x) \in N(R)[x]$.

Lemma 2.7. Given a ring $R$, the following conditions are equivalent:

(1) $R$ is nil-Armendariz and nil-IFP;

(2) $R$ is an NI ring.

Proof. $(1) \Rightarrow(2)$ Suppose that $R$ is nil-Armendariz and nil-IFP. Let $a \in N(R)$. Every element of $R a R$ is of the form $\sum_{\text {finite }}$ ras with $r, s \in R$. Every ras is nilpotent by Lemma 1.2 since $R$ is nil-IFP, and so $\sum_{\text {finite }}$ ras $\in N(R)$ by $[2$, Theorem 3.2] since $R$ is nil-Armendariz. This yields $R a R$ is nil and $a \in N^{*}(R)$, entailing that $R$ is an NI ring.

$(2) \Rightarrow(1)$ Let $R$ be NI. Then $R$ is nil-IFP clearly, and moreover $R$ is nilArmendariz by [2, Proposition 2.1].

If Köthe's conjecture does not hold, then one should find a nil-IFP ring but not NI out of the class of nil-Armendariz rings, considering Proposition 1.9(3) and Lemma 2.7.

Proposition 2.8. Let $R$ be an Armendariz ring. Then the following conditions are equivalent:

(1) $R$ is nil-IFP;

(2) $R$ is NI;

(3) $R$ is 2-primal;

(4) $R[x]$ is nil-IFP;

(5) $R[x]$ is NI;

(6) $R[x]$ is 2-primal.

Proof. Armendariz rings are nil-Armendariz by [2, Proposition 2.7]. Hence $R$ is NI by Lemma 2.7 if $R$ is nil-IFP. If $R$ is Armendariz and NI, then $R[x]$ is NI by [16, Proposition 20]. If $R$ is Armendariz, then $N_{*}(R)=N^{*}(R)$ by [17, Lemma 2.3(5)], but since $R$ is NI we have $N_{*}(R)=N^{*}(R)=N(R)$. This implies that $R$ is 2-primal, and so $R[x]$ is 2-primal by [6, Proposition 2.6]. The proof of the remainder is obtained by Lemma 1.3(1).

The condition Armendariz in Proposition 2.8 is not superfluous since there exist many NI rings but not 2-primal by [14, Example 1.2], Marks [23, Example $2.2]$ and Smoktunowicz [28]. 


\section{References}

[1] D. D. Anderson and V. Camillo, Armendariz rings and Gaussian rings, Comm. Algebra 26 (1998), no. 7, 2265-2272.

[2] R. Antoine, Nilpotent elements and Armendariz rings, J. Algebra 319 (2008), no. 8, 3128-3140.

[3] E. P. Armendariz, A note on extensions of Baer and P.P.-rings, J. Austral. Math. Soc. 18 (1974), 470-473.

[4] E. P. Armendariz, H. K. Koo, and J. K. Park, Isomorphic Ore extensions, Comm. Algebra 15 (1987), no. 12, 2633-2652.

[5] H. E. Bell, Near-rings in which each element is a power of itself, Bull. Austral. Math. Soc. 2 (1970), 363-368.

[6] G. F. Birkenmeier, H. E. Heatherly, and E. K. Lee, Completely prime ideals and associated radicals, Ring theory (Granville, OH, 1992), 102-129, World Sci. Publ., River Edge, NJ, 1993.

[7] V. Camillo and P. P. Nielsen, McCoy rings and zero-divisors, J. Pure Appl. Algebra 212 (2008), no. 3, 599-615.

[8] K. E. Eldridge, Orders for finite noncommutative rings with unity, Amer. Math. Monthly 73 (1968), 512-514.

[9] K. R. Goodearl, Von Neumann Regular Rings, Pitman, London, 1979.

[10] K. R. Goodearl and R. B. Warfield, Jr., An Introduction to Noncommutative Noetherian Rings, Cambridge University Press, 1989.

[11] I. N. Herstein, Topics in Ring Theory, The University of Chicago Press, Chicago-London, 1969.

[12] C. Y. Hong and T. K. Kwak, On minimal strongly prime ideals, Comm. Algebra 28 (2000), no. 10, 4867-4878.

[13] C. Huh, H. K. Kim, and Y. Lee, Questions on 2-primal rings, Comm. Algebra 26 (1998), no. 2, 595-600.

[14] S. U. Hwang, Y. C. Jeon, and Y. Lee, Structure and topological conditions of NI rings, J. Algebra 302 (2006), no. 1, 186-199.

[15] Y. C. Jeon, Insertion-of-idempotents-property and abelian rings, submitted.

[16] N. K. Kim and Y. Lee, Nil-Armendariz rings and upper nilradicals, submitted.

[17] N. K. Kim, K. H. Lee, and Y. Lee, Power series rings satisfying a zero divisor property, Comm. Algebra 34 (2006), no. 6, 2205-2218.

[18] A. A. Klein, Rings of bounded index, Comm. Algebra 12 (1984), no. 1-2, 9-21.

[19] R. L. Kruse and D. T. Price, Nilpotent Rings, Gordon and Breach, New York, London, Paris, 1969.

[20] J. Lambek, On the representation of modules by sheaves of factor modules, Canad. Math. Bull. 14 (1971), 359-368.

[21] Lectures on Rings and Modules, Blaisdell Publishing Company, Waltham, 1966.

[22] T. K. Lee and Y. Q. Zhou, Armendariz and reduced rings, Comm. Algebra 32 (2004), no. 6, 2287-2299.

[23] G. Marks, On 2-primal Ore extensions, Comm. Algebra 29 (2001), no. 5, 2113-2123.

[24] _ A taxonomy of 2-primal rings, J. Algebra 266 (2003), no. 2, 494-520.

[25] L. Motais de Narbonne, Anneaux semi-commutatifs et unis riels anneaux dont les id aux principaux sont idempotents, Proceedings of the 106th National Congress of Learned Societies (Perpignan, 1981), Bib. Nat., Paris (1982), 71-73.

[26] M. B. Rege and S. Chhawchharia, Armendariz rings, Proc. Japan Acad. Ser. A Math. Sci. 73 (1997), no. 1, 14-17.

[27] G. Shin, Prime ideals and sheaf representation of a pseudo symmetric ring, Trans. Amer. Math. Soc. 184 (1973), 43-60.

[28] A. Smoktunowicz, Polynomial rings over nil rings need not be nil, J. Algebra 233 (2000), no. $2,427-436$. 
JINEON BAEK

Department of Mathematics

Korea Science Academy of KAIST

Pusan 614-103, Korea

WoOyoung Chin

Department of Mathematics

Korea ScIEnce ACAdemy of KAIST

PusAn 614-103, Korea

JiWOONG CHOI

Department of Mathematics

Korea Science ACAdemy of KAIST

Pusan 614-103, Korea

TAEHYUN EOM

Department of Mathematics

Korea Science Academy of KAIST

Pusan 614-103, KoreA

Young CHeOl JeON

Department of Mathematics

Korea ScIEnce ACAdemy of KAIST

PusAn 614-103, Korea

E-mail address: ycjeon@kaist.ac.kr

YANG LEE

Department of Mathematics

Pusan National University

Pusan 609-735, Korea

E-mail address: ylee@pusan.ac.kr 